\title{
The interplay between Campylobacter and Helicobacter species and other gastrointestinal microbiota of commercial broiler chickens
}

\author{
Nadeem O Kaakoush ${ }^{1}$, Nidhi Sodhi ${ }^{1}$, Jeremy W Chenu ${ }^{2}$, Julian M Cox ${ }^{1,3}$, Stephen M Riordan ${ }^{4,5}$ and Hazel M Mitchell ${ }^{{ }^{*}}$
}

\begin{abstract}
Background: Poultry represent an important source of foodborne enteropathogens, in particular thermophilic Campylobacter species. Many of these organisms colonize the intestinal tract of broiler chickens as harmless commensals, and therefore, often remain undetected prior to slaughter. The exact reasons for the lack of clinical disease are unknown, but analysis of the gastrointestinal microbiota of broiler chickens may improve our understanding of the microbial interactions with the host.
\end{abstract}

Methods: In this study, the fecal microbiota of 31 market-age (56-day old) broiler chickens, from two different farms, was analyzed using high throughput sequencing. The samples were then screened for two emerging human pathogens, Campylobacter concisus and Helicobacter pullorum, using species-specific PCR.

Results: The gastrointestinal microbiota of chickens was classified into four potential enterotypes, similar to that of humans, where three enterotypes have been identified. The results indicated that variations between farms may have contributed to differences in the microbiota, though each of the four enterotypes were found in both farms suggesting that these groupings did not occur by chance. In addition to the identification of Campylobacter jejuni subspecies doylei and the emerging species, C. concisus, C. upsaliensis and H. pullorum, several differences in the prevalence of human pathogens within these enterotypes were observed. Further analysis revealed microbial taxa with the potential to increase the likelihood of colonization by a number of these pathogens, including C. jejuni.

Conclusion: Depletion of these taxa and the addition of taxa that compete with these pathogens, may form the basis of competitive exclusion strategies to eliminate them from the gastrointestinal tract of chickens.

Keywords: Broiler chicken, Gastrointestinal tract, Microbiota, Pathogen, Campylobacter, Helicobacter, Gallibacterium, Campylobacter concisus

\section{Background}

Contaminated poultry products are a major source of human foodborne acute bacterial gastroenteritis pathogens worldwide [1]. Epidemiological studies indicate that between $50-80 \%$ of all human Campylobacter infections are attributed to poultry, in particular the handling and consumption of raw or undercooked broiler chicken meat [1]. Given that the majority of warm-blooded domestic animals as well as wild animals and birds shed viable Campylobacter species in their feces, it is not surprising

\footnotetext{
* Correspondence: H.Mitchell@unsw.edu.au

'School of Biotechnology and Biomolecular Sciences, The University of New South Wales, Sydney, NSW 2052, Australia

Full list of author information is available at the end of the article
}

that these organisms are abundant on poultry farms and in the surrounding environment [2]. Colonization of broiler flocks with Campylobacter species typically occurs between 2-3 weeks of age and Campylobacter-positive birds often remain colonized until slaughter [3]. Moreover, although high levels (up to $10^{9}$ bacteria/g) of Campylobacter spp. have been recovered from the ceca, chickens appear to suffer no clinical or other adverse effects [3]. More recently, other emerging Campylobacter and the closely related Helicobacter species have been detected within the microbiota of chickens and on processed chicken meat [4,5], suggesting that chickens may also serve as a reservoir for these species, and thus, may also be transmitted to humans. 
The annual costs associated with campylobacteriosis are extremely high, estimated to be $\$ 1.7$ billion in the United States alone [6]. Given the association between chickens and campylobacteriosis, as well as the high costs associated with this disease, many countries have investigated intervention strategies to reduce or eliminate Campylobacter from the chicken meat primary production and processing chain [7]. Such intervention strategies not only address the spread of Campylobacter species on farms and the surrounding environment, but also strategies aimed at reducing the bacterial load of Campylobacter spp. in the intestinal tract of infected chickens or increasing the resistance of chickens to Campylobacter carriage [3,8].

While intervention strategies are currently being em ployed to limit the transmission of Campylobacter species on farms and their surrounding environment, adherence to these strategies differs considerably among farms. Based on the current literature the use of hygiene barriers at the entrance to poultry houses, the provision of hand washing facilities, boot dips, house specific boots and overshoes have all been shown to prevent Campylobacter colonization of chicks $[2,3,9]$. Other strategies that have been developed to combat the bacterial load of Campylobacter spp. in the intestinal tract of infected chickens include vaccination and the use of bacteriocins, bacteriophages and probiotics $[10,11]$. Such approaches have been reported to lead to a reduction in intestinal colonization levels of broiler chickens and in some cases have resulted in a considerable decline in human campylobacteriosis rates [12]. Indeed, a quantitative microbial risk assessment determined that a reduction of $2.0 \log _{10}$ Campylobacter cells per broiler carcass would result in a 30-fold decline in human campylobacteriosis [13].

In some countries, competitive exclusion has been successfully employed to limit the colonization of chickens with Salmonella and Escherichia coli [14,15]. Potential competitive exclusion strategies to limit the prevalence of C. jejuni have also been investigated, with some degree of success [16-18]. Given this, further insights into the microbiota of chickens may aid the development of competitive exclusion strategies for Campylobacter and Helicobacter species. Thus, in the current study, the fecal microbiota of 31 market-age broiler chickens were analyzed using high-throughput sequencing and PCR to determine the prevalence and relative abundance of Campylobacter and Helicobacter species, and to identify bacterial taxa that may be associated with the absence or carriage of these enteric pathogens in commercial poultry.

\section{Results and discussion}

Classification of the gastrointestinal microbiota of chickens The gastrointestinal microbiota plays an important role in the growth and development of chickens [19]. Several important human pathogens are commonly found within the chicken microbiota, though typically they are nonpathogenic to chickens [3]. As a result, chickens are one of the key reservoirs for transmission of foodborne disease. To gain a better understanding of the influence of the chicken gastrointestinal microbiota on the carriage of Campylobacter and Helicobacter species, the fecal microbiota of 31, 56-day old chickens originating from two different farms were analyzed using high throughput sequencing (average number of reads \pm SEM: $18227 \pm 1836$ ). Based on PCA, the microbiota were separated into four potential enterotypes: enterotype 1 dominated by Firmicutes, enterotype 2 by Firmicutes and Proteobacteria, enterotype 3 by Firmicutes and Actinobacteria and enterotype 4 by Firmicutes and Bacteroidetes (Figure 1). Analysis using SIMPER confirmed these groupings with the cumulative contribution of these taxa being higher than $95 \%$ for each of these enterotypes (Table 1).

Upon further analysis, it is possible that enterotypes 2 and 3 were derived from enterotype 1, with the dominant Firmicutes being Lactobacillus and Peptostreptococcaceae for all three groupings (Figure 2). Conceivably, chickens with microbiota classified as enterotype 1 were at some later point efficiently colonized by either Proteobacteria (enterotype 2) or Actinobacteria (enterotype 3), thus resulting in the shared dominance between the respective taxa. However, only studies investigating the development of the microbiota during rearing will definitively ascertain the evolution of these enterotypes. In contrast, the Firmicutes from enterotype 4 were dominated by Ruminococcaceae, and this dominance was shared with the Bacte roidetes taxa Alistipes and Bacteroides (Figure 2). One explanation for this is that colonization by Bacteroidetes resulted in a shift in dominance from Lactobacillus and Peptostreptococcaceae to Ruminococcaceae, as these bacterial groups were present in enterotypes 1, 2 and 3, albeit in lower abundance. In addition to these findings, the lack of differentiation of dominant taxa beyond the family classification suggests that, in some cases, novel taxa may be present, as has been observed previously in the gut microbiota of post-hatch broiler chickens [20]. Interestingly, the dominance of Firmicutes, in particular Lactobacillus, within the gastrointestinal microbiome of broiler chickens has been documented previously [21-24]. However, to our knowledge, the possible grouping of the chicken microbiota into four different enterotypes and the differences in their composition has not been previously reported.

To investigate these enterotype groupings further, the microbial diversity within the 31 samples was examined. Enterotypes 1, 2 and 4 had a similar average number of species and species richness, and this was significantly lower than that of enterotype 3 (ANOVA, $P<0.005$ ) (Table 2). The diversity (Shannon index, $\mathrm{H}^{\prime}$ ) of enterotypes 3 and 4 were the highest among the enterotypes, reflecting the high species richness in enterotype 3 (Table 2), the 

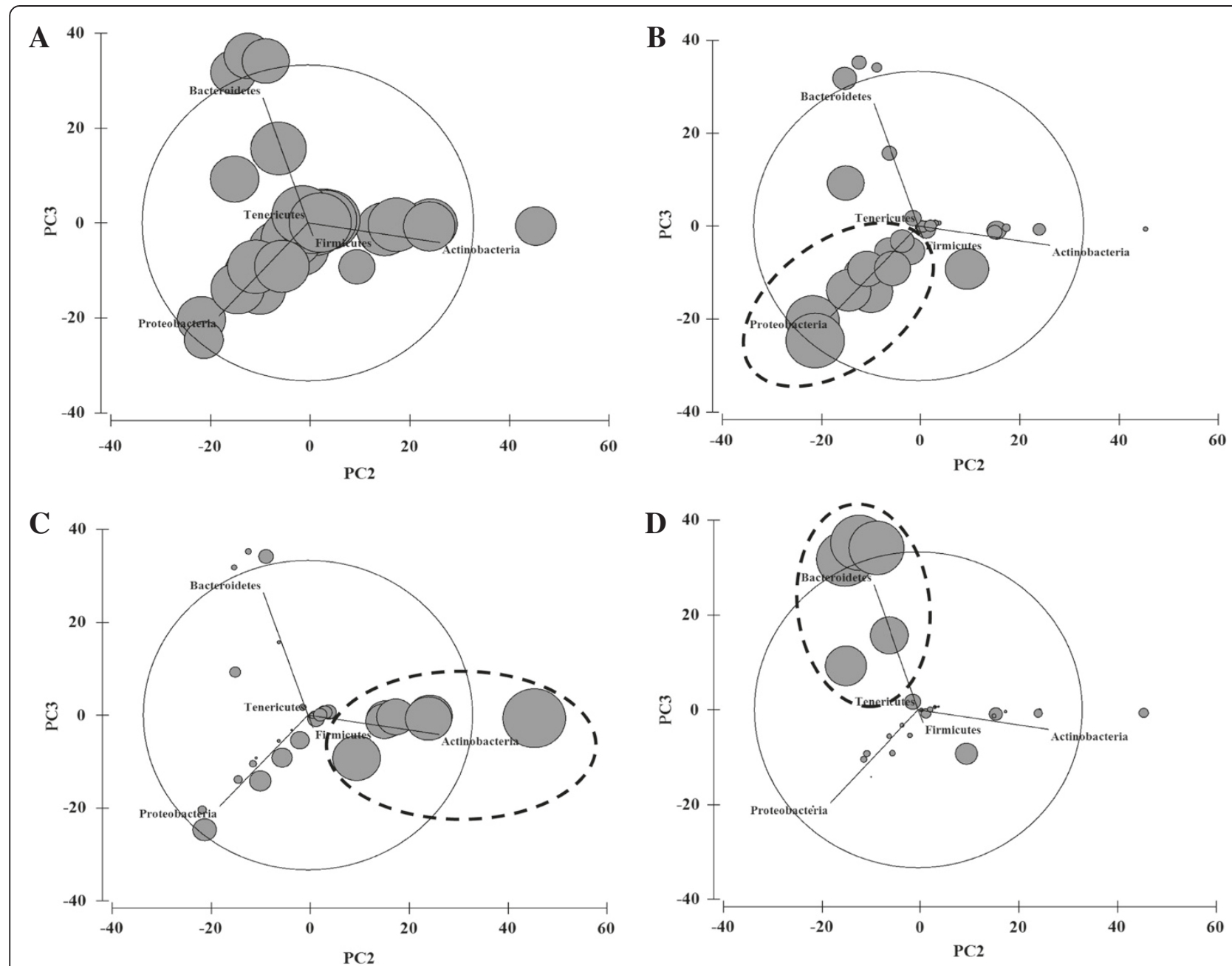

Figure 1 Principal component analysis of the $\mathbf{3 1}$ chicken microbiota samples. Circles reflect the abundance of Firmicutes (A), Proteobacteria (B), Actinobacteria (C), and Bacteroidetes (D). Dotted lines encompass samples differentiated into enterotype 2 (B), 3 (C) and 4 (D). Enterotype 1 samples aggregate in the center and cannot be differentiated along these axes.

higher contribution of the non-Firmicutes taxa within both of these enterotypes (Table 1) and the different composition of Firmicutes within enterotype 4 (Figure 2). In contrast, the diversity for enterotype 1 was significantly lower than the other three enterotypes $(P<0.0001)$, most probably due to the extensive dominance of Firmicutes.

\section{Effect of farming practices on broiler chicken} gastrointestinal microbiota

Both broiler farms used in the study belong to the same vertically integrated commercial broiler chicken operation and are located within a $25 \mathrm{~km}$ radius of each other. Farm 1 operates as a conventional (indoor barn) housing system,

Table 1 SIMPER analysis of the cumulative contribution of taxa to the overall microbiota of the enterotype

\begin{tabular}{lllllll}
\hline Enterotype & Phylum & Average abundance & Average similarity & Similarity/SD & Contribution (\%) & Cumulative contribution (\%) \\
\hline 1 & Firmicutes & 95.23 & 93.69 & 32.17 & 97.50 & 97.50 \\
2 & Firmicutes & 71.24 & 62.53 & 4.34 & 77.02 & 77.02 \\
& Proteobacteria & 24.97 & 17.41 & 2.26 & 21.45 & 98.47 \\
3 & Firmicutes & 61.09 & 51.09 & 3.01 & 66.15 & 66.15 \\
& Actinobacteria & 31.34 & 24.43 & 5.23 & 31.64 & 97.79 \\
& Firmicutes & 58.44 & 53.78 & 22.41 & 64.76 & 64.96 \\
\end{tabular}




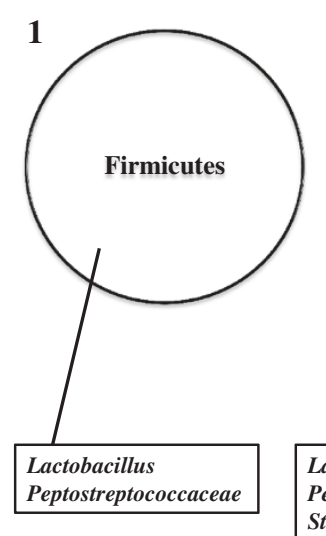

2

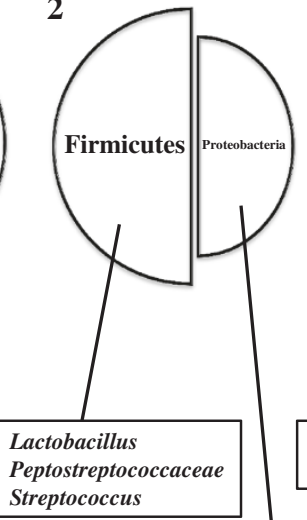

Escherichia Shigella

Enterobacter
3

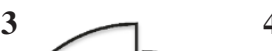

4

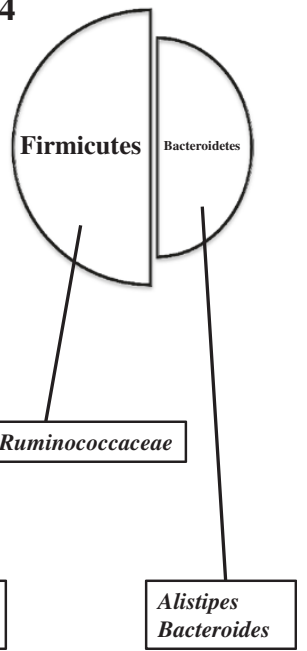

Figure 2 Taxa identified to be the highest contributors within each enterotype.

whereas farm 2 practices a free-range (extensive) system. The key differences between the two management practices is that free-range flocks are allowed access to outdoor areas, are stocked at lower densities $\left(16-32 \mathrm{~kg} / \mathrm{m}^{2}\right.$ vs $28-$ $40 \mathrm{~kg} / \mathrm{m}^{2}$ ), and the therapeutic and/or prophylactic use of antibiotics is prohibited. Samples collected from both broiler farms were found to contain all four enterotypes (Table 2). Interestingly, no significant differences in the number of species (Farm 1: 127 vs. Farm 2: 126, $P=0.95$ ), species richness (Farm 1: 27.4 vs. Farm 2: 26.4, $P=0.79$ ) or species diversity (Farm 1: 2.715 vs. Farm 2: $2.342, P=$ 0.095) were found between farms; however, chickens from farm 1 appeared to be predominantly classified under enterotype 2 and 4, reflecting an increased dominance of Gram-negative bacteria. Although the number of samples analyzed from farm 1 was low and our knowledge of the identity and quantity of the administered antibiotics limited, these findings raise the possibility that farming practices, particularly antibiotic use, may influence the identity of the taxa within the gastrointestinal microbiota of broiler chickens, but not the species richness. These findings are of particular interest given that Torok and colleagues have reported an OTU assigned to Enterobacteriaceae to be less prevalent in the gut of chicks raised on an antimicrobialfree diet and OTUs assigned to a range of Gram-positive Firmicutes, including Lachnospiraceae, Lactobacillus johnsonii, Ruminococcaceae and Oxalobacteraceae, to be less prevalent in the guts of chicks fed antimicrobialsupplemented diets [20].

\section{Differences between the chicken and human gastrointestinal microbiota}

Based on our own findings and those of others [25,26], there is a clear difference in the composition of the chicken and human gastrointestinal microbiota. In chickens the highest contributors have been identified as Lactobacillus (average abundance $=35.05 \%$ ) and Peptostreptococcaceae (average abundance $=19.48 \%$ ). However, in humans, while Firmicutes also dominate the microbiota, the taxa identified to play a major role include Blautia, Roseburia, Faecalibacterium, Ruminococcus, Coprococcus, Clostridium, Subdoligranulum and Bacteroides (Table 3) [27,28]. The importance of these differences requires further investigation, as potentially such differences, in addition to other physiological factors such as core body temperature and cell surface receptors, may explain why chickens infected with Campylobacter and other human pathogens do not develop clinical disease.

\section{Prevalence of Campylobacter, Helicobacter and Gallibacterium species in chickens}

The prevalence of Campylobacter, Helicobacter and Gallibacterium species were also determined in the 31 chicken samples using the pyrosequencing data (Table 2). This showed that C. jejuni subsp. jejuni was detected in $41.9 \%$, Campylobacter jejuni subsp. doylei in $61.3 \%$, C. concisus in $6.4 \%$, Campylobacter upsaliensis in $9.7 \%, H$. pullorum in $22.6 \%$, Helicobacter brantae in $64.5 \%$ and Gallibacterium anatis in $54.8 \%$ of chicken samples (Table 2). Given that pyrosequencing is less sensitive than species-specific PCR, it is likely that this approach may underestimate the prevalence of some of these species.

The prevalence of both $C$. jejuni subspecies were observed to be lower in enterotype 3 than in the other enterotypes, suggesting that Actinobacteria may compete with these bacteria within the gastrointestinal tract. Similarly, the dominance of Bacteroidetes appeared to have a negative effect on $G$. anatis, as this bacterium was absent 
Table 2 Microbial diversity and pathogen prevalence in 31 chicken samples determined using pyrosequencing

\begin{tabular}{|c|c|c|c|c|c|c|c|c|c|c|c|c|}
\hline Enterotype & Sample & Farm & Species & Species richness & $\mathbf{H}^{\prime}$ & $C_{j j}$ & Cjd & Cco & Cup & $\mathrm{Hpu}$ & $\mathrm{Hbr}$ & Gal \\
\hline \multirow[t]{9}{*}{1} & 1 & 1 & 169 & 36.50 & 2.248 & - & + & + & - & - & + & + \\
\hline & 13 & 2 & 150 & 17.60 & 2.309 & - & - & - & - & - & - & - \\
\hline & 16 & 2 & 85 & 18.24 & 1.185 & - & - & - & - & - & - & - \\
\hline & 23 & 2 & 132 & 28.45 & 1.874 & + & + & - & + & - & + & + \\
\hline & 24 & 2 & 127 & 27.36 & 2.350 & - & - & - & - & - & - & + \\
\hline & 25 & 2 & 104 & 22.37 & 1.661 & + & + & - & - & - & + & + \\
\hline & 27 & 2 & 87 & 18.67 & 2.053 & - & - & - & - & - & - & - \\
\hline & 28 & 2 & 133 & 28.66 & 1.506 & + & + & - & + & + & + & + \\
\hline & 29 & 2 & 118 & 25.41 & 2.282 & + & + & - & - & - & + & + \\
\hline Average & & & 123 & 24.81 & 1.940 & & & & & & & \\
\hline Prevalence & & & & & & 44.4 & 55.6 & 11.1 & 22.2 & 11.1 & 55.6 & 66.7 \\
\hline \multirow[t]{10}{*}{2} & 4 & 1 & 97 & 20.85 & 2.049 & + & + & - & + & - & + & + \\
\hline & 6 & 1 & 113 & 24.82 & 2.970 & + & + & - & - & - & + & - \\
\hline & 8 & 1 & 200 & 43.21 & 2.701 & + & + & - & - & + & + & + \\
\hline & 9 & 1 & 139 & 29.97 & 2.987 & + & - & - & - & - & + & + \\
\hline & 10 & 1 & 23 & 4.78 & 2.490 & - & - & - & - & - & - & - \\
\hline & 17 & 2 & 102 & 21.93 & 2.413 & - & + & - & - & - & + & + \\
\hline & 20 & 2 & 71 & 15.20 & 1.659 & - & - & - & - & - & - & - \\
\hline & 21 & 2 & 107 & 23.02 & 1.598 & - & + & - & - & + & + & + \\
\hline & 26 & 2 & 92 & 19.76 & 2.434 & - & + & - & - & + & + & + \\
\hline & 31 & 2 & 162 & 34.96 & 2.632 & + & + & + & - & - & + & + \\
\hline Average & & & 111 & 23.85 & 2.390 & & & & & & & \\
\hline Prevalence & & & & & & 50.0 & 70.0 & 10.0 & 10.0 & 30.0 & 80.0 & 70.0 \\
\hline \multirow[t]{7}{*}{3} & 2 & 1 & 186 & 40.17 & 3.244 & - & - & - & - & - & - & - \\
\hline & 11 & 2 & 129 & 27.79 & 2.327 & - & - & - & - & - & - & + \\
\hline & 14 & 2 & 156 & 33.66 & 2.751 & + & + & - & - & - & + & + \\
\hline & 15 & 2 & 130 & 28.01 & 2.518 & - & - & - & - & - & - & + \\
\hline & 18 & 2 & 167 & 36.05 & 2.758 & - & - & - & - & - & - & - \\
\hline & 19 & 2 & 213 & 46.04 & 3.236 & - & + & - & - & - & + & + \\
\hline & 30 & 2 & 185 & 39.96 & 3.791 & + & + & - & - & - & + & - \\
\hline Average & & & 167 & 35.95 & 2.950 & & & & & & & \\
\hline Prevalence & & & & & & 28.6 & 42.9 & 0 & 0 & 0 & 42.9 & 57.1 \\
\hline \multirow[t]{5}{*}{4} & 3 & 1 & 91 & 19.54 & 2.570 & + & + & - & - & - & + & - \\
\hline & 5 & 1 & 112 & 24.10 & 2.830 & + & + & - & - & + & + & - \\
\hline & 7 & 1 & 140 & 30.18 & 3.059 & - & + & - & - & + & + & - \\
\hline & 12 & 2 & 150 & 32.35 & 3.015 & - & + & - & - & + & + & - \\
\hline & 22 & 2 & 46 & 9.77 & 2.835 & - & - & - & - & - & - & - \\
\hline Average & & & 108 & 23.19 & 2.860 & & & & & & & \\
\hline Prevalence & & & & & & 40.0 & 80.0 & 0 & 0 & 60.0 & 80.0 & 0 \\
\hline Total prevalence & & & & & & 41.9 & 61.3 & 6.4 & 9.7 & 22.6 & 64.5 & 54.8 \\
\hline
\end{tabular}

$\mathrm{H}^{\prime}$ is the Shannon index; Cjj is C. jejuni subsp. jejuni, Cjd is C. jejuni subsp. doylei; Cco is C. concisus; Cup is C. upsaliensis; Hpu is Helicobacter pullorum; Hbr is Helicobacter brantae; and Gal: Gallibacterium anatis. 
Table 3 SIMPER analysis of the cumulative contribution of taxa to the overall microbiota of the human gastrointestinal tract

\begin{tabular}{llllll}
\hline Genus & Average abundance & Average similarity & Similarity/SD & Contribution (\%) & Cumulative contribution (\%) \\
\hline Blautia & 15.68 & 9.51 & 2.02 & 17.44 & 17.44 \\
Roseburia & 12.76 & 9.12 & 2.22 & 16.72 & 34.16 \\
Faecalibacterium & 13.65 & 7.76 & 1.16 & 14.22 & 48.37 \\
Ruminococcus & 9.72 & 5.6 & 1.45 & 10.26 & 58.63 \\
Coprococcus & 7.08 & 5.54 & 2.99 & 10.15 & 68.78 \\
Clostridium & 6.84 & 5.13 & 2.78 & 9.41 & 78.19 \\
Subdoligranulum & 5.53 & 4.06 & 2.61 & 7.44 & 85.63 \\
Bacteroides & 8.91 & 3.03 & 0.57 & 5.55 & 91.18
\end{tabular}

Results were obtained from the analysis of 20 healthy controls previously analyzed by our group [27]. Percentage cut-off for contributing taxa was $90 \%$.

in enterotype 4. To confirm these trends, further studies using a higher number of samples from all enterotypes should be analyzed and the application of species-specific PCR performed. C. jejuni subsp. doylei was detected at high prevalence in fecal samples from these chickens. This bacterium causes both gastritis and enteritis; however, it is more commonly isolated from blood cultures than stool cultures from human patients [29]. Interestingly, over a five-year period, C. jejuni subsp. doylei was isolated from 85.2\% of Campylobacter/Helicobacter-related bacteremia cases in Australia [29].

In the current study, $H$. brantae was also detected at high prevalence in these broiler fecal specimens. This urease-negative Helicobacter was first isolated from Canada geese within the greater Boston area [30]; however, little is known about the pathogenesis of this bacterium. In addition to the above species, two emerging pathogens $C$. concisus and C. upsaliensis [31,32] were detected at low prevalence in these chickens (Table 2), suggesting that chickens may act as a reservoir of emerging Campylobacter species. Lynch et al. have previously reported the detection and isolation of these fastidious Campylobacter species from chicken meat [4], indicating that, similar to the closely related $C$. jejuni, these species can remain viable following processing.

\section{Influence of Campylobacter, Helicobacter and}

Gallibacterium on the composition of the chicken gastrointestinal microbiota

The microbial composition of the chicken gastrointestinal tract was then analyzed with respect to the presence of these bacteria, independent of the enterotype of the chickens. Correlation analysis was performed between each of the genera Campylobacter, Helicobacter and Gallibacterium against all other genera detected in these samples. This showed that the abundance of Helicobacter correlated with that of Ureaplasma ( $r=0.58,95 \%$ CI: 0.28-0.77, $P=0.0006)$, though the significance of this correlation is unclear.
We then employed SIMPER to determine the effect of these pathogens on other taxa within the microbiota (Additional file 1). For each pathogen we identified the taxa that adhered to the following four conditions: 1) Presence within the 95\% contribution cut-off, 2) contribution to the similarity between the positive samples, 3) contribution to the dissimilarity between the positive and negative samples, and 4) the lack of contribution to the similarity of the negative samples. For C. jejuni, these taxa were Escherichia, Alistipes, Enterococcus, Bacteroides, Shigella, Gallibacterium, Campylobacter, Faecalibacterium, Blautia, Enterobacter and Clostridium. Moreover, the presence of $C$. jejuni was also associated with a lower abundance of Lactobacillus (32.2\% vs. $40.3 \%$ ) and Corynebacterium (2.0\% vs. $8.9 \%$ ) and a higher abundance of both Streptococcus (4.4\% vs. 1.9\%) and Ruminococcaceae (5.8\% vs. 3.3\%). Interestingly, Bereswill et al. have recently reported that, in humans, a diet-induced alteration of the intestinal microbiota, comprising an increase in the abundance of E. coli and Eubacterium spp. and a decrease in Enterococcus and Lactobacillus spp., was associated with a greater susceptibility to $C$. jejuni infection [33]. This is in line with our findings that showed that Lactobacillus spp. were lower in abundance and Escherichia was a major contributor in chickens colonized with $C$. jejuni. Moreover, three other identified taxa, Bacteroides, Alistipes and Blautia, are all major producers of short-chain fatty acids (SCFA). Given that $C$. jejuni can utilize acetate and lactate as carbon sources and these SCFAs contribute to $C$. jejuni colonization of the gut [34], it is possible that SCFAs produced by these genera could potentially serve as energy sources for $C$. jejuni and influence its ability to colonize the chicken gastrointestinal tract. In addition to this, four of the taxa (Faecalibacterium, Blautia, Clostridium and Bacteroides) that have been found to be major contributors to the human gastrointestinal microbiota were also associated with the presence of $C$. jejuni in the chicken gastrointestinal tract.

Similarly, SIMPER analysis for $H$. pullorum identified Bacteroides, Alistipes, Faecalibacterium, Coprococcus, 
Blautia, Gallibacterium, Clostridium, Pseudobutyrivibrio, Sutterella, Megamonas, Roseburia, Campylobacter, Anaerotruncus, Helicobacter and Barnesiella to be taxa of interest. As observed with $C$. jejuni, the presence of $H$. pullorum was associated with a lower abundance of Lactobacillus (21.8\% vs. $38.9 \%)$ and Corynebacterium (0.66\% vs. $5.5 \%)$ and a higher abundance of Streptococcus (7.6\% vs. $2.3 \%$ ) and Ruminococcaceae (9.3\% vs. 3.6\%). In contrast, Gallibacterium was the only taxon identified to satisfy the four conditions for the $H$. brantae positive samples. In addition, $H$. brantae was associated with a lower abundance of Ruminococcaceae (2.6\% vs. $8.1 \%$ ) and higher abundance of Peptostreptococcaceae (22.8\% vs. $14.9 \%$ ) and Corynebacterium (6.4\% vs. $1.7 \%$ ).

No taxa were identified that adhered to the four conditions for either C. upsaliensis or G. anatis, though the presence of either of these taxa was associated with substantially higher levels of Lactobacillus (51.2\% vs. 33.3\% and $42.5 \%$ vs. $26.0 \%$, respectively) and Peptostreptococcaceae $(38.0 \%$ vs. $17.5 \%$ and $25.6 \%$ vs. $12.1 \%$, respectively). Such differences may reflect the effect of these bacterial species on the host microbiota, or alternatively, their growth requirements and ability to compete for nutrients within the gastrointestinal tract.

\section{Prevalence of Campylobacter concisus and Helicobacter pullorum in chickens}

To date there have been a limited number of studies on the prevalence of emerging Campylobacter and Helicobacter species in chickens $[4,5]$. Given that $C$. concisus and H. pullorum were identified in these chickens, the prevalence of these bacteria using a more sensitive approach (species-specific PCR) was further investigated. Based on PCR, C. concisus and $H$. pullorum were shown to be present in $22.6 \%$ and $32.2 \%$ of chicken samples, respectively (Table 4), confirming that chickens have the potential to act as reservoirs for these species.

SIMPER analysis revealed Campylobacter to be the only taxon of interest in C. concisus-positive samples (Additional file 2). However, similar to C. upsaliensis, C. concisus was associated with higher levels of Lactobacillus (44.0\% vs. 32.4\%) and Peptostreptococcaceae (29.1\% vs. 16.7\%), suggesting that these emerging Campylobacter species are more prevalent in a gastrointestinal tract substantially dominated by Firmicutes.

SIMPER analysis showed $H$. pullorum prevalence to be associated with similar taxa to those previously identified, including Bacteroides, Alistipes, Faecalibacterium, Coprococcus, Blautia, Clostridium, Gallibacterium, Pseudobutyrivibrio, Sutterella, Atopostipes and Megamonas (Additional file 2). Similar to the previous analysis, the presence of $H$. pullorum was associated with a lower abundance of Lactobacillus (30.1\% vs. 37.4\%) and a higher abundance of Streptococcus (6.0\% vs. $2.4 \%$ ) and Ruminococcaceae $(7.0 \%$ vs. $3.9 \%)$. In contrast, in the new analysis, the prevalence of
Table 4 Prevalence of Campylobacter concisus and Helicobacter pullorum in 31 chicken samples determined using pyrosequencing and PCR

\begin{tabular}{lllll}
\hline Enterotype & Sample & Farm & Cco & Hpu \\
\hline 1 & 1 & 1 & + & + \\
13 & 2 & - & - \\
16 & 2 & - & - \\
23 & 2 & + & - \\
24 & 2 & - & + \\
25 & 2 & + & - \\
27 & 2 & - & - \\
28 & 2 & - & + \\
29 & 2 & + & -
\end{tabular}

Prevalence

2 (20)

$\begin{array}{ll}3 & 1 \\ 5 & 1 \\ 7 & 2 \\ 12 & 2 \\ 22 & \end{array}$

\begin{tabular}{ll}
- & - \\
- & + \\
- & + \\
- & + \\
- & - \\
0 & 60.0 \\
22.6 & 32.2 \\
\hline
\end{tabular}

Cco is C. concisus; and Hpu is Helicobacter pullorum.

Corynebacterium was found to be higher in the $H$. pullorum-positive samples (6.2\% vs. $3.6 \%)$.

\section{Conclusions}

In the current study we have shown that the gastrointestinal microbiota of chickens could be classified into potential 
enterotypes, which is consistent with the detection of three enterotypes in humans [35]. While variations between farms may have contributed to differences in prevalence of specific enterotypes, the identification of each of the enterotypes on both farms suggests that these groupings were not Coincidental. A number of differences in the prevalence of Campylobacter and Helicobacter species within these enterotypes were found, which provides possible insights into the microbial taxa that may increase the likelihood of colonization by these pathogens. Depletion of these taxa and addition of competitive taxa has the potential to aid the development of competitive exclusion strategies to eliminate these pathogens from the gastrointestinal tract of chickens. Further studies assessing larger sample numbers of fecal specimens collected over time to account for fluctuations in the microbiota [36] and screening the lower and upper intestines and ceca of broiler chickens are required to confirm our findings.

\section{Methods}

\section{Sampling and extraction protocol}

Freshly voided chicken feces were aseptically collected into $70 \mathrm{ml}$ fecal specimen containers (S5744F, TechnoPlas; St Marys, SA, Australia) from two broiler farms in the Sydney Basin region just prior to depopulation (flock age of 56 days). Samples were transported to the testing facility at $4^{\circ} \mathrm{C}$ and stored at $-20^{\circ} \mathrm{C}$ until analysis. DNA was extracted using the method of Griffiths and colleagues [37]. The concentration and quality of DNA was measured using a Nanodrop ND-1000 Spectrophotometer (Nanodrop Technologies; Wilmington, USA).

\section{Tag-encoded FLX amplicon pyrosequencing}

The microbial community was assessed by high-thro ughput sequencing of the 16S rRNA gene. Tag-encoded FLX amplicon pyrosequencing (bTEFAP) was performed as described previously [38-42] using the primers Gray28F (5' TTTGATCNTGGCTCAG) and Gray519r (5' GTNTTA CNGCGGCKGCTG) numbered in relation to E. coli $16 \mathrm{~S}$ rRNA (variable regions $1-3$ ). The sequence of the primers is not complementary to chicken DNA, thus preventing coamplification of chicken sequences. Moreover, the primers span the variable region of the rRNA gene so that discrimination between closely related taxa can be performed. At the same time, the positioning of the primers allows for amplification of a large proportion of known 16S rRNA sequences. Generation of the sequencing library utilized a one-step PCR with a total of 30 cycles, a mixture of Hot Start and HotStar high fidelity Taq polymerases, and amplicons originating and sequencing extending from the $28 \mathrm{~F}$ position with an average read length of $400 \mathrm{bp}$. Tagencoded FLX amplicon pyrosequencing analyses utilized a Roche 454 FLX instrument with Titanium reagents. This
bTEFAP process was performed at the Molecular Research LP laboratory (MR DNA; Shallowater, TX) based upon established and validated protocols.

\section{Data analysis}

The sequence data derived from the high-throughput sequencing process was analyzed employing a pipeline developed at Molecular Research LP. Sequences are first depleted of barcodes and primers, then short sequences $(<200 \mathrm{bp})$, sequences with ambiguous base calls, and sequences with homopolymer runs exceeding 6 bp are all removed. Sequences were then de-noised and chimeras were removed (Black Box Chimera Check software B2C2) [43]. Operational taxonomic units (OTU) were defined after removal of singleton sequences (sequences appearing only once in the whole dataset) with clustering set at 3\% divergence (97\% similarity) [38-42]. OTUs were then taxonomically classified using BLASTn against a curated GreenGenes database [44] and compiled into each taxonomic level. Taxonomy was defined based on the following percentages: >97\%, species; between 97\% and 95\%, unclassified species; between $95 \%$ and $90 \%$, unclassified genus; between $90 \%$ and $85 \%$, unclassified family; between $85 \%$ and $80 \%$, unclassified order; between $80 \%$ and $77 \%$, unclassified phylum; $<77 \%$, unclassified. Principal component analyses (PCA), diversity analyses (DIVERSE) and analysis of similarities and species contributions (SIMPER) based on relative abundances of bacterial groups were performed using Primer-E [45].

\section{Detection of Campylobacter concisus and Helicobacter pullorum}

Campylobacter concisus DNA was amplified using a nested PCR procedure with the first step employing Campylobacter genus-specific primers, C412F and C1228R [46] and the thermal cycling conditions $94^{\circ} \mathrm{C}$ for $5 \mathrm{~min}$, 40 cycles of $94^{\circ} \mathrm{C}$ for $10 \mathrm{~s}, 55^{\circ} \mathrm{C}$ for $10 \mathrm{~s}$, and $72^{\circ} \mathrm{C}$ for $45 \mathrm{~s}$, followed by $72^{\circ} \mathrm{C}$ for $7 \mathrm{~min}$. The second amplification utilized the C. concisus-specific primers: 5'-CTT GTG AAA TCC TAT GGC TTA- 3' (Concisus F) and 5'-CTC ATT AGA GTG CTC AGC C-3' (Concisus R), which were previously optimized by Man et al. [47]. Cycling conditions were 40 cycles of $94^{\circ} \mathrm{C}$ for $10 \mathrm{~s}, 65^{\circ} \mathrm{C}$ for $10 \mathrm{~s}$, and $72^{\circ} \mathrm{C}$ for $30 \mathrm{~s}$. Helicobacter pullorum DNA was amplified using $H$. pullorum-specific primers targeting the $16 \mathrm{~S}$ rDNA gene as previously described [48].

\section{Additional files}

Additional file 1: Contribution of taxa to the similarity and dissimilarity between subgroups of chicken. Variables analyzed were the presence of C. jejuni, C. upsaliensis, H. pullorum, H. brantae and G. anatis derived from Table 2 . The number of $C$. concisus positive samples 
was below the required threshold of 3 samples for statistical analysis. Percentage cut-off for contributing taxa was $95 \%$.

Additional file 2: Contribution of taxa to the similarity and dissimilarity between subgroups of chicken. Variables analyzed were the presence of $C$. concisus and $H$. pullorum derived from Table 3. Percentage cut-off for contributing taxa was 95\%.

\section{Competing interests}

No conflicts of interest exist.

\section{Authors' contributions}

HMM, JMC, JWC and NOK conceived the idea; HMM, SMR, JMC, JWC contributed reagents; JWC collected and organized all chicken samples; NS extracted and prepared all DNA samples; NOK performed the microbial community analyses; NS performed the species-specific PCRs; NOK, NS, HMM JWC, JMC and SMR analysed the results; NOK, HMM, JWC, JMC and SMR drafted the manuscript. All authors read and approved the final manuscript.

\section{Acknowledgements}

NOK is supported by an Early Career fellowship from the National Health and Medical Research Council, Australia. NOK and HMM would like to acknowledge financial support from The University of New South Wales.

\section{Author details}

${ }^{1}$ School of Biotechnology and Biomolecular Sciences, The University of New South Wales, Sydney, NSW 2052, Australia. ${ }^{2}$ Birling Avian Laboratories, Bringelly, NSW 2556, Australia. ${ }^{3}$ Faculty of Science, The University of New South Wales, Sydney, NSW 2052, Australia. ${ }^{4}$ Gastrointestinal and Liver Unit, The Prince of Wales Hospital, Randwick, NSW 2031, Australia. ${ }^{5}$ Prince of Wales Clinical School, The University of New South Wales, Sydney, NSW 2052, Australia.

Received: 7 May 2014 Accepted: 30 May 2014

Published: 4 June 2014

\section{References}

1. European Food Safety Authority: Scientific opinion on Campylobacter in broiler meat production: control options and performance objectives and/or targets at different stages of the food chain. EFSA J 2011, 9:2105.

2. Ellis-Iversen J, Ridley A, Morris V, Sowa A, Harris J, Atterbury R, Sparks N, Allen $\mathrm{V}$ : Persistent environmental reservoirs on farms as risk factors for Campylobacter in commercial poultry. Epidemiol Infect 2012, 140:916-924.

3. Newell DG, Elvers KT, Dopfer D, Hansson I, Jones P, James S, Gittins J, Stern NJ, Davies R, Connerton I, Pearson D, Salvat G, Allen VM: Biosecurity-based interventions and strategies to reduce Campylobacter spp. on poultry farms. Appl Environ Microbiol 2011, 77:8605-8614.

4. Lynch OA, Cagney C, McDowell DA, Duffy G: Occurrence of fastidious Campylobacter spp. in fresh meat and poultry using an adapted cultural protocol. Int J Food Microbiol 2011, 150:171-177.

5. Manfreda G, Parisi A, Lucchi A, Zanoni RG, De Cesare A: Prevalence of Helicobacter pullorum in conventional, organic, and free-range broilers and typing of isolates. Appl Environ Microbiol 2011, 77:479-484

6. Batz MB, Hoffmann S, Morris JG Jr: Ranking the disease burden of 14 pathogens in food sources in the United States using attribution data from outbreak investigations and expert elicitation. J Food Prot 2012, 75:1278-1291.

7. Wagenaar JA, French NP, Havelaar AH: Preventing Campylobacter at the source: why is it so difficult? Clin Infect Dis 2013, 57:1600-1606.

8. Havelaar AH, van Pelt W, Ang CW, Wagenaar JA, van Putten JP, Gross U, Newell DG: Immunity to Campylobacter: its role in risk assessment and epidemiology. Crit Rev Microbiol 2009, 35:1-22.

9. Cox JM, Pavic A: Advances in enteropathogen control in poultry production. J Appl Microbiol 2010, 108:745-755.

10. Annamalai T, Pina-Mimbela R, Kumar A, Binjawadagi B, Liu Z, Renukaradhya GJ, Rajashekara G: Evaluation of nanoparticle-encapsulated outer membrane proteins for the control of Campylobacter jejuni colonization in chickens. Poult Sci 2013, 92:2201-2211.

11. Ghareeb K, Awad WA, Mohnl M, Porta R, Biarnes M, Bohm J, Schatzmayr G: Evaluating the efficacy of an avian-specific probiotic to reduce the colonization of Campylobacter jejuni in broiler chickens. Poult Sci 2012 91:1825-1832.

12. Lammerding AM, Fazil A: Hazard identification and exposure assessment for microbial food safety risk assessment. Int J Food Microbio/ 2000, 58:147-157.

13. Rosenquist $H$, Nielsen NL, Sommer HM, Norrung B, Christensen BB: Quantitative risk assessment of human campylobacteriosis associated with thermophilic Campylobacter species in chickens. Int J Food Microbiol 2003, 83:87-103.

14. Kerr AK, Farrar AM, Waddell LA, Wilkins W, Wilhelm BJ, Bucher O, Wills RW, Bailey $\mathrm{RH}$, Varga C, McEwen SA, Rajic A: A systematic review-meta-analysis and meta-regression on the effect of selected competitive exclusion products on Salmonella spp. prevalence and concentration in broiler chickens. Prev Vet Med 2013, 111:112-125.

15. Nuotio L, Schneitz C, Nilsson O: Effect of competitive exclusion in reducing the occurrence of Escherichia coli producing extendedspectrum beta-lactamases in the ceca of broiler chicks. Poult Sci 2013, 92:250-254.

16. Lin J: Novel approaches for Campylobacter control in poultry. Foodborne Pathog Dis 2009, 6:755-765.

17. Neal-McKinney JM, Lu X, Duong T, Larson CL, Call DR, Shah DH, Konkel ME: Production of organic acids by probiotic lactobacilli can be used to reduce pathogen load in poultry. PLoS One 2012, 7:e43928.

18. Zhang G, Ma L, Doyle MP: Potential competitive exclusion bacteria from poultry inhibitory to Campylobacter jejuni and Salmonella. J Food Prot 2007, 70:867-873

19. Yeoman CJ, Chia N, Jeraldo P, Sipos M, Goldenfeld ND, White BA: The microbiome of the chicken gastrointestinal tract. Anim Health Res Rev 2012, 13:89-99.

20. Torok VA, Allison GE, Percy NJ, Ophel-Keller K, Hughes RJ: Influence of antimicrobial feed additives on broiler commensal posthatch gut microbiota development and performance. Appl Environ Microbiol 2011, 77:3380-3390

21. Danzeisen JL, Kim HB, Isaacson RE, Tu ZJ, Johnson TJ: Modulations of the chicken cecal microbiome and metagenome in response to anticoccidial and growth promoter treatment. PLOS One 2011, 6:e27949.

22. Qu A, Brulc JM, Wilson MK, Law BF, Theoret JR, Joens LA, Konkel ME, Angly F, Dinsdale EA, Edwards RA, Nelson KE, White BA: Comparative metagenomics reveals host specific metavirulomes and horizontal gene transfer elements in the chicken cecum microbiome. PLoS One 2008 3:e2945.

23. Rehman HU, Vahjen W, Awad WA, Zentek J: Indigenous bacteria and bacterial metabolic products in the gastrointestinal tract of broiler chickens. Arch Anim Nutr 2007, 61:319-335.

24. Stanley D, Geier MS, Hughes RJ, Denman SE, Moore RJ: Highly variable microbiota development in the chicken gastrointestinal tract. PLoS One 2013, 8:e84290.

25. Lee JE, Lee $\mathrm{S}$, Sung J, Ko G: Analysis of human and animal fecal microbiota for microbial source tracking. ISME J 2011, 5:362-365.

26. Lei $F$, Yin $Y$, Wang $Y$, Deng B, Yu HD, Li L, Xiang C, Wang S, Zhu B, Wang $X$ Higher-level production of volatile fatty acids in vitro by chicken gut microbiotas than by human gut microbiotas as determined by functional analyses. Appl Environ Microbiol 2012, 78:5763-5772.

27. Kaakoush NO, Day AS, Huinao KD, Leach ST, Lemberg DA, Dowd SE, Mitchell HM: Microbial dysbiosis in pediatric patients with Crohn's disease. J Clin Microbiol 2012, 50:3258-3266.

28. Walker AW, Duncan SH, Louis P, Flint HJ: Phylogeny, culturing, and metagenomics of the human gut microbiota. Trends Microbiol 2014, 22:267-274.

29. Parker CT, Miller WG, Horn ST, Lastovica AJ: Common genomic features of Campylobacter jejuni subsp. doylei strains distinguish them from C. jejuni subsp. jejuni. BMC Microbiol 2007, 7:50.

30. Fox JG, Taylor NS, Howe S, Tidd M, Xu S, Paster BJ, Dewhirst FE: Helicobacter anseris sp. nov. and Helicobacter brantae sp. nov., isolated from feces of resident Canada geese in the greater Boston area. Appl Environ Microbiol 2006, 72:4633-4637.

31. Kaakoush NO, Mitchell HM: Campylobacter concisus - A new player in intestinal disease. Front Cell Infect Microbiol 2012, 2:4.

32. Man SM: The clinical importance of emerging Campylobacter species. Nat Rev Gastroenterol Hepatol 2011, 8:669-685.

33. Bereswill S, Plickert R, Fischer A, Kuhl AA, Loddenkemper C, Batra A, Siegmund B, Gobel UB, Heimesaat MM: What you eat is what you get: 
Novel Campylobacter models in the quadrangle relationship between nutrition, obesity, microbiota and susceptibility to infection. Eur J Microbiol Immunol 2011, 1:237-248.

34. Masanta WO, Heimesaat MM, Bereswill S, Tareen AM, Lugert R, Gross U, Zautner AE: Modification of intestinal microbiota and its consequences for innate immune response in the pathogenesis of campylobacteriosis. Clin Dev Immunol 2013, 2013:526860.

35. Arumugam M, Raes J, Pelletier E, Le Paslier D, Yamada T, Mende DR, Fernandes GR, Tap J, Bruls T, Batto JM, Bertalan M, Borruel N, Casellas F, Fernandez L, Gautier L, Hansen T, Hattori M, Hayashi T, Kleerebezem M, Kurokawa K, Leclerc M, Levenez F, Manichanh C, Nielsen HB, Nielsen T, Pons N, Poulain J, Qin J, Sicheritz-Ponten T, Tims S, et al: Enterotypes of the human gut microbiome. Nature 2011, 473:174-180.

36. Sekelja M, Rud I, Knutsen SH, Denstadli V, Westereng B, Næs T, Rudi K: Abrupt temporal fluctuations in the chicken fecal microbiota are explained by its gastrointestinal origin. Appl Environ Microbiol 2012, 78:2941-2948

37. Griffiths RI, Whiteley AS, O'Donnell AG, Bailey MJ: Rapid method for coextraction of DNA and RNA from natural environments for analysis of ribosomal DNA- and rRNA-based microbial community composition. Appl Environ Microbiol 2000, 66:5488-5491.

38. Andreotti R, de Leon AA P, Dowd SE, Guerrero FD, Bendele KG, Scoles GA: Assessment of bacterial diversity in the cattle tick Rhipicephalus (Boophilus) microplus through tag-encoded pyrosequencing. BMC Microbiol 2011, 11:6.

39. Bailey MT, Dowd SE, Parry NM, Galley JD, Schauer DB, Lyte M: Stressor exposure disrupts commensal microbial populations in the intestines and leads to increased colonization by Citrobacter rodentium. Infect Immun 2010, 78:1509-1519.

40. Dowd SE, Callaway TR, Wolcott RD, Sun Y, McKeehan T, Hagevoort RG, Edrington TS: Evaluation of the bacterial diversity in the feces of cattle using $16 \mathrm{~S}$ rDNA bacterial tag-encoded FLX amplicon pyrosequencing (bTEFAP). BMC Microbiol 2008, 8:125.

41. Dowd SE, Delton Hanson J, Rees E, Wolcott RD, Zischau AM, Sun Y, White J, Smith DM, Kennedy J, Jones CE: Survey of fungi and yeast in polymicrobial infections in chronic wounds. J Wound Care 2011, 20:40-47.

42. Dowd SE, Sun Y, Wolcott RD, Domingo A, Carroll JA: Bacterial tag-encoded FLX amplicon pyrosequencing (bTEFAP) for microbiome studies: bacterial diversity in the ileum of newly weaned Salmonella-infected pigs. Foodborne Pathog Dis 2008, 5:459-472.

43. Gontcharova V, Youn E, Wolcott RD, Hollister EB, Gentry TJ, Dowd SE: Black Box Chimera Check (B2C2): a Windows-Based Software for Batch Depletion of Chimeras from Bacterial 16S rRNA Gene Datasets. Open Microbiol J 2010, 4:47-52.

44. DeSantis TZ, Hugenholtz P, Larsen N, Rojas M, Brodie EL, Keller K, Huber T, Dalevi D, Hu P, Andersen GL: Greengenes, a chimera-checked 16S rRNA gene database and workbench compatible with ARB. Appl Environ Microbiol 2006, 72:5069-5072.

45. Clarke KR: Non-parametric multivariate analyses of changes in community structure. Austr J Ecol 1993, 18:117-143.

46. Linton D, Owen RJ, Stanley J: Rapid identification by PCR of the genus Campylobacter and of five Campylobacter species enteropathogenic for man and animals. Res Microbiol 1996, 147:707-718.

47. Man SM, Zhang L, Day AS, Leach ST, Lemberg DA, Mitchell H: Campylobacter concisus and other Campylobacter species in children with newly diagnosed Crohn's disease. Inflamm Bowel Dis 2010, 16:1008-1016.

48. Stanley J, Linton D, Burnens AP, Dewhirst FE, On SL, Porter A, Owen RJ Costas M: Helicobacter pullorum sp. nov.-genotype and phenotype of a new species isolated from poultry and from human patients with gastroenteritis. Microbiology 1994, 140:3441-3449.

doi:10.1186/1757-4749-6-18

Cite this article as: Kaakoush et al:: The interplay between

Campylobacter and Helicobacter species and other gastrointestinal microbiota of commercial broiler chickens. Gut Pathogens 2014 6:18.

\section{Submit your next manuscript to BioMed Central and take full advantage of:}

- Convenient online submission

- Thorough peer review

- No space constraints or color figure charges

- Immediate publication on acceptance

- Inclusion in PubMed, CAS, Scopus and Google Scholar

- Research which is freely available for redistribution

Submit your manuscript at www.biomedcentral.com/submit
C Biomed Central 